\title{
CRITERIA FOR REGIONALLY RECURRENT FLOWS
}

\author{
SABER ELAYDI
}

\begin{abstract}
Regionally recurrent and $P$-regionally recurrent flows are characterized in purely prolongational notions. An example is given to show that the condition $x \in J(x)$ is not, as many authors asserted, equivalent to regional recurrence.
\end{abstract}

Introduction. Let $(X, T)$ be a flow, where $X$ is a topological space and $T$ is a topological group. Then a point $x \in X$ is said to be $T$-regionally recurrent or simply regionally recurrent if for each neighbourhood $U$ of $x$ there exists an extensive subset $A$ of $T$ such that $U \cap U a \neq \emptyset$ for all $a \in A[6]$. In [3] the author showed that for a flow $(X, T)$, a point $x \in X$ is regionally recurrent if and only if $x \in J^{P}(x)$ for all replete semigroups $P$ in $T$. An example is provided (Example 14) to show that the condition $x \in J(x)$ is not equivalent to regional recurrence as it was stated in [7] and [8]. However, in the setting of continuous flows, where the phase group is the additive group of real numbers $R$, a necessary and a sufficient condition for a point $x \in X$ to be regionally recurrent is either one of the following (1) $x \in J^{+}(x)$ or, (2) $x \in J^{-}(x)$ or (3) $x \in J(x)$. This is due to the simple structure of replete semigroups in $R$. Every replete semigroup in $R$ contains either a ray in $R^{+}$or a ray in $R^{-}$. Thus if $x \in J^{+}(x)$, then $x \in J^{P}(x)$ for all replete semigroups $P$ in $R^{+}$. Furthermore, $x \in J^{+}(x)$ implies that $x \in J^{-}(x)$. Consequently, $x \in J^{Q}(x)$ for all replete semigroups $Q$ in $R^{-}$and hence $x$ is regionally recurrent. Similar analysis is available for the cases $x \in J^{-}(x)$ and $x \in J(x)$. The situation is far more complicated in general flows as is shown in Example 14. In this example we have $x \in J(x)$ for each $x \in X$ but $x \notin J^{Q}(x)$ for some replete semigroups $Q$ in the phase group $T$.

In [3] it was shown that $x \in X$ is $P$-regionally recurrent iff it is $P$-nonwandering. The same conclusion holds for $T$-regionally recurrent and $T$-nonwandering. Hence, the work here is a natural generalization of the work in [1] on nonwandering continuous flows. However, our techniques are different and much simpler.

In addition to the above-mentioned, one purpose of this note is to introduce prolongational techniques which are widely used in Dynamical Systems Theory but surprisingly unknown in the theory of Topological Dynamics. As far as I know it was Hajek [7] who suggested the use of prolongational techniques in Topological Dynamics. R. Knight [8, 9] and Elaydi [2-5], with Kaul followed suit. But the power of these techniques has yet to be shown. Our main references for notations and terminology are [7] and [3].

Received by the editors April 6, 1981 and, in revised form, September 20, 1981.

1980 Mathematics Subject Classification. Primary 54H20.

Key words and phrases. Regional recurrence, replete semigroups, prolongation, extensive set, nonwandering. 
From now on $(X, T)$ denotes a flow, where $X$ is a Hausdorff topological space and $T$ is an abelian topological group. $P$ and $P$ will denote the set of all replete semigroups in $T$ and a certain replete semigroup in $T$, respectively.

Definition 1. A subset $A$ of $T$ is $P$-extensive if $A=S \cap P$ for some extensive set $S$ of $T$.

LEMMA 2. The following statements are pairwise equivalent for a subset $A$ of $P$.

(1) $A$ is $P$-extensive.

(2) $A \cap Q \neq \emptyset$ for all $Q \in P$ and $Q \subset P$.

(3) $A \cap p Q \neq \emptyset$ for all $Q \in P, Q \subset P$ and $p \in P$.

Proof. (1) implies (2). Since $A$ is $P$-extensive, $A=S \cap P$ for some extensive set $S$ in $T$. Hence $S \cap Q \neq \emptyset[6,6.15]$. This implies that $A \cap Q=S \cap P \cap Q=$ $S \cap Q \neq 0$.

(2) implies (3). Let $p \in P$. Let $K$ be a compact subset of $T$ and let $H=p^{-1} K$. Then there exists $t \in T$ such that $H t=t p^{-1} K \subset Q$. It follows that $t K \subset p Q$. Hence $p Q$ is a replete semigroup in $T$ which is contained in $P$. Hence $A \cap p Q \neq \emptyset$.

(3) implies (1). Define $S=A \cap(T-P)$. Then clearly $A=S \cap P$. Furthermore, $S$ is an extensive set in $T$.

DEFINITION 3. A point $x \in X$ is said to be $P$-regionally recurrent if for each neighbourhood $U$ of $x$ there exists a $P$-extensive set $A$ such that $U \cap U a \neq \emptyset$ for all $a \in A$.

A point $x \in X$ is $T$-regionally recurrent or simply regionally recurrent if it is $P$-regionally recurrent for all $P \in P$. A transformation group $(X, T)$ is said to have one of the above properties if each point in $X$ has the property.

THEOREM 4. For any flow $(X, T)$ the following statements are pairwise equivalent.

(1) The flow is $P$-regionally recurrent.

(2) $x \in J^{Q}(x)$ for all $Q \in \mathcal{P}, Q \subset P$ and $x \in X$.

(3) $J^{Q}(x)=D^{Q}(x)$ for all $Q \in P, Q \subset P$ and $x \in X$.

(4) $D^{Q}(x)=D^{Q^{-1}}(x)$ for all $Q \in P, Q \subset P$ and $x \in X$.

Proof. (1) implies (2). Let $Q \in P$ such that $Q \subset P$ and let $U$ be a neighbourhood of $x \in X$. Then $U \cap U a \neq \emptyset$ for all $a \in A$, for some $p$-extensive set $A$. Then by Lemma 2 for each $q \in Q$ there exists $a \in A \cap q Q$. Hence $x \in \overline{U q Q}$ for each $q \in Q$. This implies that $x \in J^{Q}(x)$.

(2) implies (3). Since $J^{Q}(x)$ is invariant, $x Q \subset J^{Q}(x)$. Hence $D^{Q}(x)=x Q \cup$ $J^{Q}(x)=J^{Q}(x)$.

(3) implies (4). Let $y \in D^{Q^{-1}}(x)$. Then $x \in D^{Q}(y)=J^{Q}(y)$. This implies that $y \in J^{Q^{-1}}(x)$. Thus $J^{Q^{-1}}(x)=D^{Q^{-1}}(x)$. Let $z \in D^{Q}(x)=J^{Q}(x)$. Then $z Q^{-1} \subset J^{Q}(x)$. If $z \notin J^{Q^{-1}}(x)$, then $z Q^{-1} \cap J^{Q^{-1}}(x)=z Q^{-1} \cap D^{Q^{-1}}(x)=\emptyset$ which is clearly absurd. Hence $D^{Q}(x)=D^{Q^{-1}}(x)$.

(4) implies (1). Let $x \in X$ and $Q \in P$ with $Q \subset P$. Since $x Q \subset D^{Q}(x)=$ $D^{Q^{-1}}(x)$, for each $q \in Q$ we have $x q^{-1} \in D^{Q}(x)=D^{Q^{-1}}(x)$. This implies that $x \in D^{Q^{-1}}(x) q=D^{Q^{-1}}(x q)=D^{Q}(x q)$ for all $q \in Q$. Thus for each neighbourhood $U$ of $x$ and $q \in Q$ we have $x \in \overline{U q Q}$. For each $q \in Q$ choose $b \in q Q$ such that $U \cap U b \neq \emptyset$. Let $A$ be the set of all such elements $b$ with the above-mentioned properties ranging over all replete semigroups $Q$ contained in $P$. Then according to 
Lemma 2 the set $A$ is $P$-extensive. Consequently $x$ is $P$-regionally recurrent. This completes the proof of the theorem.

COROLlARY 5. For any flow $(X, T)$ the following statements are pairwise equivalent.

(1) The flow $(X, T)$ is regionally recurrent.

(2) $x \in J^{P}(x)$ for each $x \in X$ and $P \in P$.

(3) $J^{P}(x)=D^{P}(x)$ for each $x \in X$ and $P \in P$.

(4) $D^{P}(x)=D^{P^{-1}}(x)$ for each $x \in X$ and $P \in P$.

LEMMA 6. Let $\left\{x_{i}\right\}$ and $\left\{y_{i}\right\}$ be nets in $X$ such that $x_{i} \rightarrow x$ and $y_{i} \rightarrow y$. If for each $i, y_{i} \in J^{P}\left(x_{i}\right)$ then $y \in J^{P}(x)$.

PROOF. The proof follows immediately from [2, 2.7].

THEOREM 7. A flow $(X, T)$ is p-regionally recurrent iff the set of p-regionally recurrent points in $X$ is dense in $X$.

PROOF. This follows from Lemma 6 and Theorem 4.

COROLLARY 8. A flow $(X, T)$ is regionally recurrent iff the set of regionally recurrent points in $X$ is dense in $X$.

LEMMA 1.9. If $y \in L^{P}(x)$ for $P \in P$, then $J^{P}(x) \subset J^{P}(y)=D^{P}(y)$.

Proof. Let $z \in J^{P}(x)$. Since $J^{P}(x p)=J^{P}(x)$ for all $p \in P, z \in J^{P}(x p)$ for each $p \in P$. There exists a net $x p_{i} \rightarrow y, p_{i} \in P$. It follows from Lemma 6 that $z \in J^{P}(y)$. Hence $J^{P}(x) \subset J^{P}(y)$. Furthermore, $y \in L^{P}(x) \subset J^{P}(x) \subset J^{P}(y)$ implies that $J^{P}(y)=D^{P}(y)$, since $D^{P}(y)=y P \cup J^{P}(y)=J^{P}(y)$, as $J^{P}(y)$ is invariant.

THEOREM 10. If $y \in L^{Q}(x)$ for all $Q \in P$ contained in $P$, then $y$ is p-regionally recurrent.

Proof. Use Lemma 9 and Theorem 4.

COROLLARY 11. If $y \in L^{P}(x)$ for all $P \in P$, then $y$ is regionally recurrent.

THEOREM 12. Let $(X, T)$ be a P-regionally recurrent flow. Then the set of $P$-recurrent points in $X$ is dense in $X$, provided that $T$ is generative [6].

Proof. We need only to replace $T$ by $Q$, where $Q$ is any replete semigroup contained in $P$, in the proof of $[8,1]$.

REMARK 1.13. Although the proof of $[8,1]$ uses wrong equivalences to regionally recurrence and recurrence it can be corrected by a slight modification. We will give an example of a flow in which $x \in L(x)$ for each $x \in X$ and yet it is neither regionally recurrent nor recurrent.

EXAMPLE 14. Let $(X, T)$ be a flow, where $X=R$; the set of real numbers with the usual topology and $T=R \times R$, with the operation $(a, b)+(c, d)=$ $(a+c, b+d)$, where $R$ is the additive group of real numbers. Define the action of $T$ on $X$ by $x(a, b)=x+a+b$ for all $x \in X$ and $(a, b) \in R \times R$. Let $P=$ $\{(x, y) \in R \times R \mid x \geq 0, y \leq 0\}$. Then for each $x \in X, x \in L^{P}(x) \cap L^{P^{-1}}(x)$. Let $Q=\{(x, y) \in R \times R \mid x \geq 0, y \geq 0\}$. Then for each $x \in X$ we have $x \notin L^{Q}(x)=$ 
$J^{Q}(x)$. Hence the flow is not regionally recurrent. Furthermore, $x \notin L^{S}(x)$ for each $x \in X$, where $S=\{(x, y) \in R \times R \mid x \geq 0,-x / 3 \leq y \leq 0\}$ is a replete semigroup contained in $P$. Thus the flow is not even $P$-regionally recurrent.

REMARK 15. In the above example the flow is of $P$-characteristic 0 [2]. Also the example $[\mathbf{5}, 5.1]$ is of $P$-characteristic 0 , where $P=R^{+}$, but not $P$-regionally recurrent. In the sequel we will discuss a few simple relations between these two notions.

THEOREM 16. Let $(X, T)$ be a flow of $P$-characteristic 0 . If $L^{Q^{-1}}(x) \neq \emptyset$ for all $x \in X$ and $Q \in P$ contained in $P$, then $(X, T)$ is $P$-regionally recurrent.

Proof. Use $[2,3.2]$ and Theorem 4.

COROLlARY 17. Let $(X, T)$ be a flow of $P$-characteristic 0 where $L^{P^{-1}}(x) \neq \emptyset$ for all $x \in X$ and $P \in P$. Then $(X, T)$ is regionally recurrent.

THEOREM 18. Let $(X, T)$ be a flow, where $X$ is assumed to be locally compact and $T$ is generative. Then $(X, T)$ is regionally recurrent and of $P$-characteristic 0 for all $P \in P$ iff it is pointwise almost periodic and of $Q$-characteristic 0 for some $Q \in P$.

Proof. Assume that $(X, T)$ is regionally recurrent and of $P$-characteristic 0 for all $P \in P$. Then it follows from $[2,3.4]$ and Corollary 1.5 that $x \in L^{P}(x)$ for each $x \in X$ and $P \in P$. Hence the flow $(X, T)$ is recurrent $[2,3.7]$. Now use [6, 7.05] and [2,3.5] to conclude the proof of the first part. To prove the converse assume that the flow is of $Q$-characteristic 0 for some $Q \in P$ and pointwise almost periodic. Then $\overline{x T}$ is compact and minimal for all $x \in X[6,4.10]$. We need to show that the flow is of $P$-characteristic 0 for each $P \in P$. Let $y \in D^{P}(x), x, y \in X$ and $P \in P$. Then there are nets $\left\{x_{i}\right\}$ in $X$ and $\left\{p_{i}\right\}$ in $P$ such that $x_{i} \rightarrow x$ and $x_{i} p_{i} \rightarrow y$. For each $i, x_{i} p_{i} \in \overline{x_{i} P} \subset L^{Q^{-1}}(x) \subset D^{Q^{-1}}(x)$. It follows from [2, 2.7] that $y \in D^{Q^{-1}}(x)=D^{Q}(x)=\overline{x Q}=\overline{x T}=\overline{x P}$. This completes the proof of the theorem.

\section{REFERENCES}

1. S. Ahmad and J. Sarabia, On non wandering continuous flows, Funkcial. Ekvac. 21 (1978), 193-201.

2. S. Elaydi, P-recursion and transformation groups of characteristic 0 , J. Univ. Kuwait (Science) 9 (1982) (to appear).

3. __ Weakly equicontinuous flows, Funkcial. Ekvac. 24 (1981).

4. - On equicontinuous transformation groups, Bull. Austral. Math. Soc. 24 (1981), 367-372.

5. S. Elaydi and S. K. Kaul, On characteristic 0 and locally weakly almost periodic flows, Preprint 42, Univ. of Regina, Canada, 1980.

6. W. H. Gottschalk and G. A. Hedlund, Topological dynamics, Amer. Math. Soc. Colloq. Publ., vol. 36, Amer. Math. Soc., Providence, R. I., 1955.

7. O. Hajek, Prolongations in topological dynamics, Seminar on Differential Equations and Dynamical Systems II, Lecture Notes in Math., vol. 44, Springer-Verlag, Berlin and New York, 1970, pp. 79-89.

8. R. Knight, The center of a transformation group, Proc. Conf. Nonlinear Phenomena in Math. (Univ. of Texas, Arlington, June 1980) (to appear).

9. __ Prolongationally stable transformation groups, Math. Z. 161 (1978), 189-194.

Department of Mathematics, Kuwait University, P.O. Box 5969, Kuwait 